\title{
Estudio del uso y consumo de dispositivos móviles en universitarios
}

\author{
Julio Ruiz-Palmero \\ julio@uma.es \\ University of Málaga, España \\ Ernesto Colomo-Magaña \\ ecolomo@uma.es \\ University of Málaga, España \\ Enrique Sánchez-Rivas \\ enriquesr@uma.es \\ University of Málaga, España \\ Teresa Linde-Valenzuela \\ tlinde@uma.es \\ University of Málaga, España
}

\begin{abstract}
Resumen
Los dispositivos móviles se están convirtiendo en una herramienta imprescindible en el día a día y en las relaciones entre el alumnado en los últimos años, gracias a sus usos y potencialidades. A pesar de ello, el uso excesivo e inadecuado puede conducir a síndromes de dependencia en algunos casos, un factor que se puede elevar debido a la situación de aislamiento provocada por la COVID-19. Este estudio pretende analizar el uso y abuso de las TIC en profesionales de la educación en formación, antes y después del confinamiento. Se ha utilizado un diseño longitudinal de panel con medidas pre-test y post-test, aplicando el instrumento validado MULTICAGE-TIC en una muestra de 147 estudiantes de la Facultad de Educación de la Universidad de Málaga. Los resultados reflejan un aumento significativo en todas TIC analizadas (internet, móvil, videojuegos, mensajería instantánea y redes sociales), pasando de conductas no problemáticas o consideradas de riesgo antes del confinamiento, a un uso abusivo tras el mismo en todas las escalas salvo en los videojuegos, que reflejan un uso problemático. El estudio refleja que la pandemia y las restricciones de movilidad e interacción social han incidido en el aumento del uso problemático de las TIC.
\end{abstract}

\section{Palabras claves}

Tecnologías de la Información y la Comunicación; adicción; formación del personal docente; COVID-19 


\title{
Study of the use and consumption of mobile devices in university students
}

\author{
Julio Ruiz-Palmero \\ julio@uma.es \\ University of Málaga, Spain \\ Ernesto Colomo-Magaña \\ ecolomo@uma.es \\ University of Málaga, Spain \\ Enrique Sánchez-Rivas \\ enriquesr@uma.es \\ University of Málaga, Spain \\ Teresa Linde-Valenzuela \\ tlinde@uma.es \\ University of Málaga, Spain
}

\begin{abstract}
Mobile devices are becoming an essential tool in the day-to-day and in relationships between students in recent years, thanks to their uses and potential. Despite this, excessive and inappropriate use can lead to dependence syndromes in some cases, a factor that can be increased by the isolation situation caused by COVID-19. This study aims to analyze the use and abuse of ICTs in the training of education professionals, before and after confinement. A longitudinal panel design with pre-test and post-test measures was used, applying the validated MULTICAGE-TIC instrument in a sample of 147 students from the Faculty of Education of the University of Malaga. The results reflect a significant increase in all ICT analyzed (internet, mobile, video games, instant messaging and social networks), going from non-problematic behaviors or considered risky before confinement, to abusive use after it in all scales except in video games, which reflect problematic use. The study reflects that the pandemic and restrictions on mobility and social interaction have led to an increase in the problematic use of ICT.
\end{abstract}

\section{Keywords}

Information and Communication Technology's; Adicction; Educational personnel training; COVID-19 


\section{Introducción}

En la actualidad sería imposible concebir nuestra sociedad sin Internet. La realidad pone de manifiesto el papel fundamental que la red juega en el día a día de nuestros jóvenes y adultos. En la última encuesta del Instituto Nacional de Estadística (INE, 2020) acerca del equipamiento y uso de TIC en los hogares españoles, en el $95.4 \%$ de los mismos se dispone de conexión a la Internet, habiendo en la actualidad en España más usuarios de Internet (93.2\%) que de ordenador (81.4\%). De hecho, el $99.5 \%$ de los internautas se conectan a la misma a través del teléfono móvil, siendo preciso destacar que el principal tipo de conexión a la Red, por banda ancha, es la conexión móvil a través de un dispositivo. Si analizamos para qué se utiliza Internet, la Encuesta sobre Equipamiento y Uso de Tecnologías de Información y Comunicación en los Hogares (INE, 2020) señala que las actividades más realizadas en los tres últimos meses por la población de 16 a 74 años ha sido usar servicios de mensajería instantánea, como WhatsApp $(89.5 \%$ actual frente al $85.1 \%$ en 2019 ), buscar información sobre bienes y servicios (78.3\% respecto al $72.9 \%$ en 2019 ) y telefonear o realizar videollamadas a través de Internet (un $77.7 \%$ por el $54.9 \%$ en 2019). Estos datos corroboran un aumento significativo en su uso en el 2020, siendo en el último, las videollamadas, el incremento más significativo, en más de 20 puntos respecto al año anterior.

El contexto que definen los datos expuestos explica los cambios producidos en las formas de comunicación y socialización, en la búsqueda y localización de información e, incluso, en las actividades desarrolladas durante el tiempo libre (Brennan, \& Dempsey, 2018). Una de las tareas más usuales desarrolladas por los jóvenes en Internet es la monitorización de redes sociales (González-Cortés, 2020), que están consideradas desde 2012 como el principal medio de información de los jóvenes (Catalina et al., 2015; Devine, \& Lloyd, 2012; García-Jiménez et al., 2018).

En la actualidad existen diferentes estudios acerca del uso de Internet que inciden, principalmente, en temas relacionados con problemas sobre su utilización, destacando la adicción a Internet (Ahmadi, 2014, Carbonell et al., 2012, Cho et al., 2014, Jasso Medrano et al., 2017). Estos trabajos relacionan dicha adicción con la reducción de la actividad física, el aislamiento y la ansiedad, introduciendo así en las investigaciones factores psicológicos, biológicos y sociales. Además, en otros estudios se incluyen ciertas características como los síntomas de abstinencia, cambios de estado de ánimo, conflictos y recaídas (Griffiths et al. 2014). Estamos, por tanto, ante un fenómeno cuya raíz se vehicula al uso abusivo de las Tecnologías de la Información y la Comunicación (TIC), produciéndose en todas las edades y en muy diferentes ámbitos culturales (Pedrero-Pérez et al., 2018). Esta utilización problemática es patente en diferentes recursos, herramientas e instrumentos, derivadas de la dependencia que los usuarios tienen a Internet.

Así, la adicción a redes sociales se considera como un subtipo de adicción a Internet, pues comparten muchas de sus características centradas en el uso de dichas aplicaciones o sitios web (Błachnio et al., 2015; Ruiz-Palmero et al., 2019). En España, la principal actividad en Internet son las redes sociales, utilizadas por el $90.8 \%$ de los jóvenes entre 16 y 24 años (INE, 2020). Además, se ha visibilizado que las redes sociales se ocupan de promover la conducta adictiva mediante diversas estrategias para manipular emociones y comportamientos, con la finalidad de mantener conectadas a las personas usuarias (Fernández Álvarez, \& Fajardo, 2020; Orlowski, 2020). La principal herramienta para acceder a dichas redes sociales son los dispositivos móviles, que han sido adoptados por los jóvenes como una extensión propia, aumentado la comunicación y sus relaciones sociales por estos canales, pero del mismo modo provocando usos problemáticos, dependencia, etc. (Ruiz-Palmero et al. 2016). En este sentido, es preciso reseñar que la dependencia o adicción no se genera respecto al dispositivo móvil, sino a las diferentes aplicaciones a las que el móvil permite acceso. De este modo, los Smartphone se convierten en el medio para utilizar las apps que generan esos comportamientos abusivos y dependientes, no siendo en sí los teléfonos inteligentes el instrumento adictivo. Al igual que con el uso desmedido de Internet, 
también se han encontrado trabajos que vinculan una utilización excesiva de los dispositivos móviles con problemas para la salud: ansiedad, depresión, insomnio, etc. (Elhai et al., 2017; Ostovar et al, 2016).

Otro recurso tecnológico cuyo uso inapropiado genera problemas son los videojuegos. La Asociación Española de Videojuegos (AEVI) indica que el número de jugadores entre 6 y 64 años ha alcanzado los 15 millones en España, lo que supone un $42 \%$ de la población en esa franja de edad (AEVI, 2019). Junto a estos datos hay que subrayar que, en la actualidad, el número de horas medias dedicada por los españoles es de 6.7 horas semanales (AEVI, 2019), inferior si lo comparamos a los de otros países europeos como Alemania (8.3 horas), Francia (8.6 horas) o Reino Unido (11 horas). No obstante, la adicción a los mismos es una realidad patente, reflejado en su reconocimiento como trastorno metal por la Organización Mundial de la Salud desde la $72^{a}$ Asamblea Mundial de la Salud, tratándose como tal a partir del año 2022 (Organización Mundial de la Salud, 2019).

En definitiva, estamos ante una variedad de recursos TIC que ofrecen muchas posibilidades en diferentes contextos y realidades, especialmente en el campo de la educación y la formación, como han demostrado ya estudios anteriores (Martín Del Pozo, 2015; Revuelta, \& Esnaola, 2013; Sánchez-Rivas et al., 2017). Sin embargo, estás potencialidades no son incompatible con los riesgos derivados de un uso inadecuado o abusivo de los mismos. Sobre este último ámbito hemos profundizado en nuestro estudio, analizando la situación actual en universitarios y la incidencia que la pandemia generada por el COVID-19 ha tenido sobre este segmento poblacional.

La declaración del estado de alarma en España en marzo de 2020 y el confinamiento soportado en los meses siguientes a la declaración de la emergencia sanitaria (Real Decreto 463/2020) ha tenido efectos en el uso y consumo de las tecnologías en los hogares, influyendo de forma directa en el trastorno de adicción a Internet que ya se venía padeciendo en años anteriores (De la Villa Moral, \& Suárez, 2016; Tsitsika et al., 2013).

Las circunstancias en las que se vio interrumpida la docencia ante la irrupción de la pandemia, y la necesidad de pasar la enseñanza presencial de todos los niveles educativos a formato virtual, ha favorecido el incremento de horas que adolescentes y jóvenes han pasado delante de las pantallas. Según datos del INE (2020), la pandemia ha disparado el uso de internet con un millón más de usuarios que al principio de ésta, y alcanza al 95,3\% de los hogares. Sumado este hecho a que gran parte del ocio de la juventud está mediado por dispositivos digitales, ya sea por consumo de videojuegos, o bien, la comunicación entre pares vía redes sociales (Viñals Blanco et al., 2014), el tiempo de conexión ha aumentado notablemente (Hernández, 2020).

Este abuso de pantallas se ha dado para todos los miembros del hogar, aunque las consecuencias no hayan sido igual para cada uno. Estudios como el de Orte Socías et al. (2020) evidencian que durante este periodo las familias no han tenido suficientes recursos para evitar el uso inadecuado de las TIC en los más jóvenes. Además, el trabajo de Paricio del Castillo y Pando Velasco (2020) evidencia cómo la pandemia del COVID-19 se asocia con un incremento de factores de riesgo psicosociales, entre otros, el uso inapropiado y abusivo de las TIC.

Teniendo todos estos aspectos en cuenta, el presente estudio tiene como objetivo analizar el uso y abuso de las TIC en profesionales de la educación en formación. Esta investigación pretende, además, explorar si existen diferencias entre los universitarios antes y después del confinamiento causado por la COVID-19. 


\section{Material y métodos}

\section{a. Enfoque metodológico}

La investigación se centra en un estudio cuantitativo ex post facto con un diseño longitudinal de panel con medidas pre-test y post-test. De este modo, recogeremos los datos sobre problemas relacionados con el uso y abuso de las TIC a un mismo grupo específico de sujetos (preestablecidos) en dos momentos diferentes (sin manipular la variable de estudio), mediante un instrumento de medida.

\section{b. Muestra}

Se ha realizado un muestreo por conveniencia entre estudiantes de la Facultad de Ciencias de la Educación de la Universidad de Málaga, durante el curso académico 2019/2020. Los participantes se han seleccionado de forma no probabilística (intencional) en función del acceso de los investigadores a diferentes asignaturas de los grados ofertados. La muestra final la conforman 147 estudiantes, con edades comprendidas entre 19 y 34 años $(M=20.52 ; S D=1.77)$. Los principales datos sociodemográficos se recogen a continuación (Tabla 1).

\begin{tabular}{|c|c|c|c|c|c|}
\hline \multirow[b]{2}{*}{ Sexo } & \multicolumn{5}{|l|}{ Grado } \\
\hline & $\begin{array}{l}\text { Educación Infantil } \\
(\%)\end{array}$ & $\begin{array}{l}\text { Educación Primaria } \\
(\%)\end{array}$ & $\begin{array}{l}\text { Pedagogía } \\
(\%)\end{array}$ & $\begin{array}{l}\text { Educación Social } \\
(\%)\end{array}$ & Subtotal (\%) \\
\hline Mujer & $22(14.97)$ & $54(36.73)$ & $12(8.16)$ & $19(12.93)$ & $107(72.79)$ \\
\hline Hombre & $2(1.36)$ & $36(24.49)$ & $1(0.68)$ & $1(0.68)$ & $40(27.71)$ \\
\hline Subtotal & $24(16.33)$ & $90(61.22)$ & $13(8.84)$ & $20(13.61)$ & $147(100)$ \\
\hline
\end{tabular}

Tabla 1. Distribución de la muestra.

\section{c. Instrumento}

Para analizar el uso y abuso respecto a las TIC, se ha utilizado el instrumento validado MULTICAGETIC (Pedrero-Pérez et al., 2018). Se trata de un cuestionario conformado por 20 ítems, divididos en 5 escalas, que permite detectar conductas problemáticas respecto al uso de internet, móvil, videojuegos, mensajería instantánea y redes sociales (Tabla 2). Sus orígenes se sitúan en el MULTICAGE-CAD-4, un cuestionario de cribado de conductas compulsivas (Pedrero-Pérez et al., 2007) aplicado en ámbitos como la atención primaria (Garrido-Elustondo et al., 2016), las adicciones comportamentales (Estévez et al., 2015) o las adicciones a sustancias (Navas et al., 2014). En el MULTICAGE-TIC, cada escala se vincula a una TIC, compuesta cada escala por cuatro preguntas asociadas a cada conducta problema: A) valoración de exceso temporal respecto a su utilización; B) valoración respecto a un uso excesivo por parte de otros agentes; C) reconocimiento de dificultad para no realizar la conducta; D) reconocimiento de dificultad para dejar de realizar voluntariamente la conducta. Cada pregunta es respondida de forma dicotómica (si/no), realizándose la interpretación a partir de su agrupación por escalas (internet, móvil, videojuegos, mensajería instantánea y redes sociales), contabilizándose el número de respuestas afirmativas para poder valorar el nivel de uso problemático en cada TIC. Este método de interpretación deviene del cuestionario CAGE (Dhalla y Kopec, 2007), un instrumento con características psicométricas conocidas y validez diagnóstica establecida (Rodríguez-Monje et al., 2019). Entre las interpretaciones encontramos: inexistencia de problemas, con una o ninguna respuesta afirmativa; uso problemático, con dos respuestas afirmativas; abuso, con tres respuestas afirmativas; dependencia, con cuatro respuestas afirmativas. 


\begin{tabular}{|c|c|c|}
\hline Escala & Ítem & $\begin{array}{l}\text { Conducta } \\
\text { problema }\end{array}$ \\
\hline \multirow{4}{*}{ Internet } & $\begin{array}{l}\text { ¿Dedica más tiempo del que cree que debería a estar conectado a } \\
\text { Internet con objetivos distintos a los de su trabajo? }\end{array}$ & A \\
\hline & ¿Se han quejado sus familiares de las horas que dedica a Internet? & B \\
\hline & $\begin{array}{l}\text { ¿Le cuesta trabajo permanecer alejado de Internet varios días } \\
\text { seguidos? }\end{array}$ & $\mathrm{C}$ \\
\hline & $\begin{array}{l}\text { ¿Tiene problemas para controlar el impulso de conectarse a Internet } \\
\text { o ha intentado sin éxito reducir el tiempo que dedica a estar } \\
\text { conectado? }\end{array}$ & $\mathrm{D}$ \\
\hline \multirow{4}{*}{ Móvil } & $\begin{array}{l}\text { ¿Utiliza el teléfono móvil más a menudo o por más tiempo del que } \\
\text { debiera? }\end{array}$ & A \\
\hline & $\begin{array}{l}\text { ¿Le han comentado en alguna ocasión, sus familiares o amigos, que } \\
\text { utiliza mucho el móvil para hablar o enviar mensajes? }\end{array}$ & $\mathrm{B}$ \\
\hline & $\begin{array}{l}\text { Si algún día no tiene su móvil ¿se siente incómodo o como si le } \\
\text { faltara algo muy importante? }\end{array}$ & $\mathrm{C}$ \\
\hline & $\begin{array}{l}\text { ¿Ha intentado reducir el uso de su móvil sin conseguirlo de forma } \\
\text { satisfactoria? }\end{array}$ & $\mathrm{D}$ \\
\hline \multirow{4}{*}{ Videojuegos } & $\begin{array}{l}\text { ¿Dedica más tiempo del que cree que debería a jugar a la } \\
\text { videoconsola o juegos de ordenador o del móvil? }\end{array}$ & A \\
\hline & $\begin{array}{l}\text { ¿Se queja su familia de que pasa demasiado tiempo jugando con la } \\
\text { videoconsola o el ordenador o el móvil? }\end{array}$ & B \\
\hline & $\begin{array}{l}\text { ¿Le cuesta trabajo estar varios días sin usar su videoconsola o sus } \\
\text { juegos de ordenador o el móvil? }\end{array}$ & C \\
\hline & $\begin{array}{l}\text { ¿Ha intentado sin éxito reducir el tiempo que dedica a jugar con su } \\
\text { videoconsola o su ordenador o su móvil? }\end{array}$ & $D$ \\
\hline \multirow{4}{*}{$\begin{array}{l}\text { Mensajería } \\
\text { instantánea }\end{array}$} & $\begin{array}{l}\text { ¿Dedica más tiempo del que cree que debería a chatear con sus } \\
\text { contactos por WhatsApp (u otro programa similar) a través del } \\
\text { móvil? }\end{array}$ & A \\
\hline & $\begin{array}{l}\text { ¿Le han dicho sus familiares o amigos que pasa demasiado tiempo } \\
\text { chateando a través del WhatsApp (u otro programa similar)? }\end{array}$ & B \\
\hline & $\begin{array}{l}\text { ¿Le cuesta trabajo pasar tiempo sin consultar el WhatsApp (u otro } \\
\text { programa similar) para comprobar si hay nuevos mensajes? }\end{array}$ & $\mathrm{C}$ \\
\hline & $\begin{array}{l}\text { ¿Ha intentado alguna vez sin éxito reducir el tiempo que dedica al } \\
\text { WhatsApp (u otro programa similar)? }\end{array}$ & $\mathrm{D}$ \\
\hline \multirow{2}{*}{$\begin{array}{l}\text { Redes } \\
\text { cnrialac }\end{array}$} & $\begin{array}{l}\text { ¿Dedica más tiempo del que cree que debería a participar en redes } \\
\text { sociales, como Facebook, Twitter, Instagram o similares? }\end{array}$ & $A$ \\
\hline & $\begin{array}{l}\text { ¿Le han dicho sus familiares o amigos que pasa demasiado tiempo } \\
\text { consultando y comunicándose a través del Facebook (o Twitter, o } \\
\text { Instagram o similares)? }\end{array}$ & $\mathrm{B}$ \\
\hline
\end{tabular}


suciaics

¿Le cuesta trabajo pasar tiempo sin consultar el Facebook (o Twitter,

o Instagram o similares) para comprobar si hay nueva información?

C

¿Ha intentado alguna vez sin éxito reducir el tiempo que dedica al

Facebook (o Twitter, o Instagram o similares)?

Tabla 2. Escalas e ítems del instrumento MULTICAGE-TIC.

Se trata de un instrumento que cumple con la validez de contenido, tras su aplicación en estudios previos (Méndez-Gago y González-Robledo, 2018; Pedrero-Pérez et al., 2018), así como de fiabilidad, con un alfa de Cronbach superior a 0.7 para la escala del móvil, y mayor de 0.8 para el resto de escalas (Pedrero-Pérez et al., 2018). Respecto al análisis factorial confirmatorio del instrumento, la estructura interna del test con 5 escalas de 4 ítems cada una, presenta un ajuste satisfactorio (RMR $=0.012 ; \mathrm{GFI}=0.96 ; \mathrm{AGFI}=0.95 ; \mathrm{NFI}=0.94 ; \mathrm{RFI}=0.92 ; \mathrm{PGFI}=0.73 ; \mathrm{PNFI}=$ $0.79)$.

\section{d. Procedimiento}

El interés inicial de este estudio se centraba en conocer el uso y abuso de las TIC por parte de futuros profesionales de la educación en formación. Sumado a ello, la pandemia ocasionada por el COVID-19 supuso una situación excepcional no vivida anteriormente. En un contexto ya de por sí digitalizado, el confinamiento desembocó en un incremento de las interacciones de carácter virtual, tanto personales como profesionales. En el caso que nos ocupa, la Facultad de Ciencias de la Educación de la Universidad de Málaga se vio abocada, como el resto de instituciones educativas de nivel superior, a desarrollar sus procesos formativos de forma online durante el segundo cuatrimestre del curso académico 2019-2020. Considerando esta circunstancia, junto a la previsión del incremento del uso de las TIC debido al aislamiento resultante del encierro obligatorio, quisimos ahondar en conocer el impacto que la pandemia ocasionaba respecto a la utilización de las TIC. La recolección de las respuestas, aportadas de forma autónoma y voluntaria por el alumnado que quiso participar en el estudio, se realizó en febrero de 2020, al inicio del cuatrimestre (partiendo del interés inicial ya comentado), y a finales de abril de 2020, en pleno estado de alarma y confinamiento. Las respuestas fueron recopiladas mediante una encuesta online autoadministrada de forma telemática, elaborada en LimeSurvey. La elección de esta herramienta se debe a su flexibilidad y personalización del proceso, ya que permite la restricción del acceso a los potenciales participantes mediante su correo electrónico, así como la realización de recordatorios para el alumnado que aún no había participado.

\section{e. Análisis de datos}

Los análisis respecto al nivel de uso y abuso de las TIC se han desarrollado con el programa SPSS v.25. En función del objetivo planteado, se han realizado las pruebas estadísticas pertinentes. Se efectúa un análisis exploratorio de los estadísticos descriptivos de las respuestas, tanto del pre-test como del post-test, considerando el número y porcentaje de respuestas afirmativa en cada escala del test, junto con su agrupación por nivel de problemática. Tras ello, se analiza la existencia de diferencias significativas en las escalas entre el pre-test y el post-test. Para elegir la prueba adecuada para dicho análisis, se examinaron los supuestos paramétricos de homocedasticidad y normalidad (Tabla 3). El estadístico de Leven reflejó una significatividad inferior a $0.05(p \leq 0.05)$ en 4 de las 5 escalas, siendo solo compatible la escala de redes sociales con la aplicación de prueba paramétrica. Respecto a la prueba de Kolmogorov-Smirnov (con corrección de 
significatividad de Lillierfors), alcanzó un nivel de significativad inferior a $0.05(p \leq 0.05)$ en todas las escalas, no ajustándose a la normalidad. Dichos resultados obligan a descartar la realización de pruebas paramétricas, por lo que aplicamos la prueba no paramétrica $\mathrm{W}$ de Wilcoxon para la comparación de medias en muestras relacionadas.

\begin{tabular}{|c|c|c|c|c|c|}
\hline \multirow{2}{*}{ Elemento } & \multirow{2}{*}{ Momento } & \multicolumn{2}{|c|}{ Kolmogorov-Smirnov } & \multicolumn{2}{|c|}{ Levene } \\
\hline & & Estadístico & Significatividad & Estadístico & Significatividad \\
\hline \multirow{2}{*}{ Internet } & Pre-test & 0.171 & $0.000 *$ & \multirow{2}{*}{36.671} & \multirow{2}{*}{$0.000 *$} \\
\hline & Post-test & 0.453 & $0.000 *$ & & \\
\hline \multirow{2}{*}{ Móvil } & Pre-test & 0.256 & $0.000 *$ & \multirow{2}{*}{123.170} & \multirow{2}{*}{$0.000 *$} \\
\hline & Post-test & 0.524 & $0.000 *$ & & \\
\hline \multirow{2}{*}{ Videojuegos } & Pre-test & 0.441 & $0.000 *$ & \multirow{2}{*}{62.527} & \multirow{2}{*}{$0.000 *$} \\
\hline & Post-test & 0.173 & $0.000 *$ & & \\
\hline \multirow{2}{*}{$\begin{array}{l}\text { Mensajería } \\
\text { instantánea }\end{array}$} & Pre-test & 0.167 & $0.000 *$ & \multirow{2}{*}{13.557} & \multirow{2}{*}{$0.000 *$} \\
\hline & Post-test & 0.417 & $0.000 *$ & & \\
\hline \multirow{2}{*}{ Redes sociales } & Pre-test & 0.163 & $0.000 *$ & \multirow{2}{*}{0.659} & \multirow{2}{*}{0.418} \\
\hline & Post-test & 0.385 & $0.000 *$ & & \\
\hline
\end{tabular}

Tabla 3. Estadísticos descriptivos en el post-test. $*=p<.05$

\section{Resultados}

Teniendo en cuenta el objetivo de nuestro estudio, comenzamos por presentar un análisis exploratorio de los resultados descriptivos del MULTICAGE-TIC (Tabla 4) antes y durante el confinamiento. Como podemos ver, se detalla el número de preguntas respondidas afirmativas en cada escala, tanto en el pre-test como en el post-test, junto con las puntuaciones medias y desviación típica por escala. 


\begin{tabular}{|c|c|c|c|c|c|c|c|c|}
\hline \multirow{2}{*}{ Escala } & \multirow{2}{*}{ Momento } & \multicolumn{5}{|c|}{$\mathrm{N}(\%)$ de preguntas respondidas afirmativamente } & \multirow{2}{*}{ Media } & \multirow{2}{*}{$\begin{array}{l}\text { Desv. } \\
\text { típica }\end{array}$} \\
\hline & & 0 & 1 & 2 & 3 & 4 & & \\
\hline \multirow[t]{2}{*}{ Internet } & Pre-test & $11(7.48)$ & $34(23.13)$ & $50(34.01)$ & $39(26.53)$ & $13(8.85)$ & 2.06 & 1.07 \\
\hline & Post-test & $0(0)$ & $2(1.36)$ & $5(3.40)$ & $28(19.05)$ & $112(76.19)$ & 3.70 & 0.60 \\
\hline \multirow[t]{2}{*}{ Móvil } & Pre-test & $2(1.36)$ & $19(12.93)$ & $67(45.58)$ & $41(27.89)$ & $18(12.24)$ & 2.37 & 0.91 \\
\hline & Post-test & $0(0)$ & $0(0)$ & $3(2.04)$ & $11(7.48)$ & $133(90.48)$ & 3.88 & 0.38 \\
\hline \multirow[t]{2}{*}{ Videojuegos } & Pre-test & $109(74.15)$ & $17(11.56)$ & $13(8.84)$ & $8(5.45)$ & $0(0)$ & 0.46 & 0.87 \\
\hline & Post-test & $27(18.37)$ & $30(20.41)$ & $30(20.41)$ & $20(13.60)$ & $40(27.21)$ & 2.11 & 1.47 \\
\hline \multirow{2}{*}{$\begin{array}{l}\text { Mensajería } \\
\text { instántanea }\end{array}$} & Pre-test & $22(14.97)$ & $31(21.09)$ & $40(27.21)$ & $38(25.85)$ & $16(10.88)$ & 1.97 & 1.23 \\
\hline & Post-test & $1(0.68)$ & $6(4.08)$ & $17(11.56)$ & $20(13.61)$ & $103(70.07)$ & 3.48 & 0.90 \\
\hline \multirow{2}{*}{$\begin{array}{l}\text { Redes } \\
\text { sociales }\end{array}$} & Pre-test & $16(10.88)$ & $31(21.09)$ & $45(30.61)$ & $30(20.41)$ & $25(17.01)$ & 2.12 & 1.24 \\
\hline & Post-test & $4(2.72)$ & $9(6.12)$ & $25(17.01)$ & $15(10.20)$ & $94(63.95)$ & 3.27 & 1.11 \\
\hline
\end{tabular}

Tabla 4. Estadísticos descriptivos pre-test y post-test.

En cuanto a los resultados generales, se refleja un incremento en todas las escalas, aumentando las medias en un rango de 1.15 a 1.65 puntos. Este hecho refleja que la situación excepcional de aislamiento provocó que aumentara más de 1 punto de media el uso de cada una de las TIC. Cabe destacar que el móvil es el elemento con mayor uso, tanto antes $(2.37 \pm 0.91)$ como tras el confinamiento ( $3.88 \pm 0.38$ ), ocurriendo el caso contrario en los videojuegos ( $0.46 \pm 0.87$ en el pre-test, $2.11 \pm 1.47$ en el post-test).

Para conseguir un mayor nivel de profundidad en los análisis, antes de seguir comparando los resultados en ambos momentos, vamos a describir la distribución de las puntuaciones en el pretest y post-test, interpretando los resultados según el nivel de problemática reflejado en casa escala. Comenzamos por los resultados del pre-test (Figura 1). 


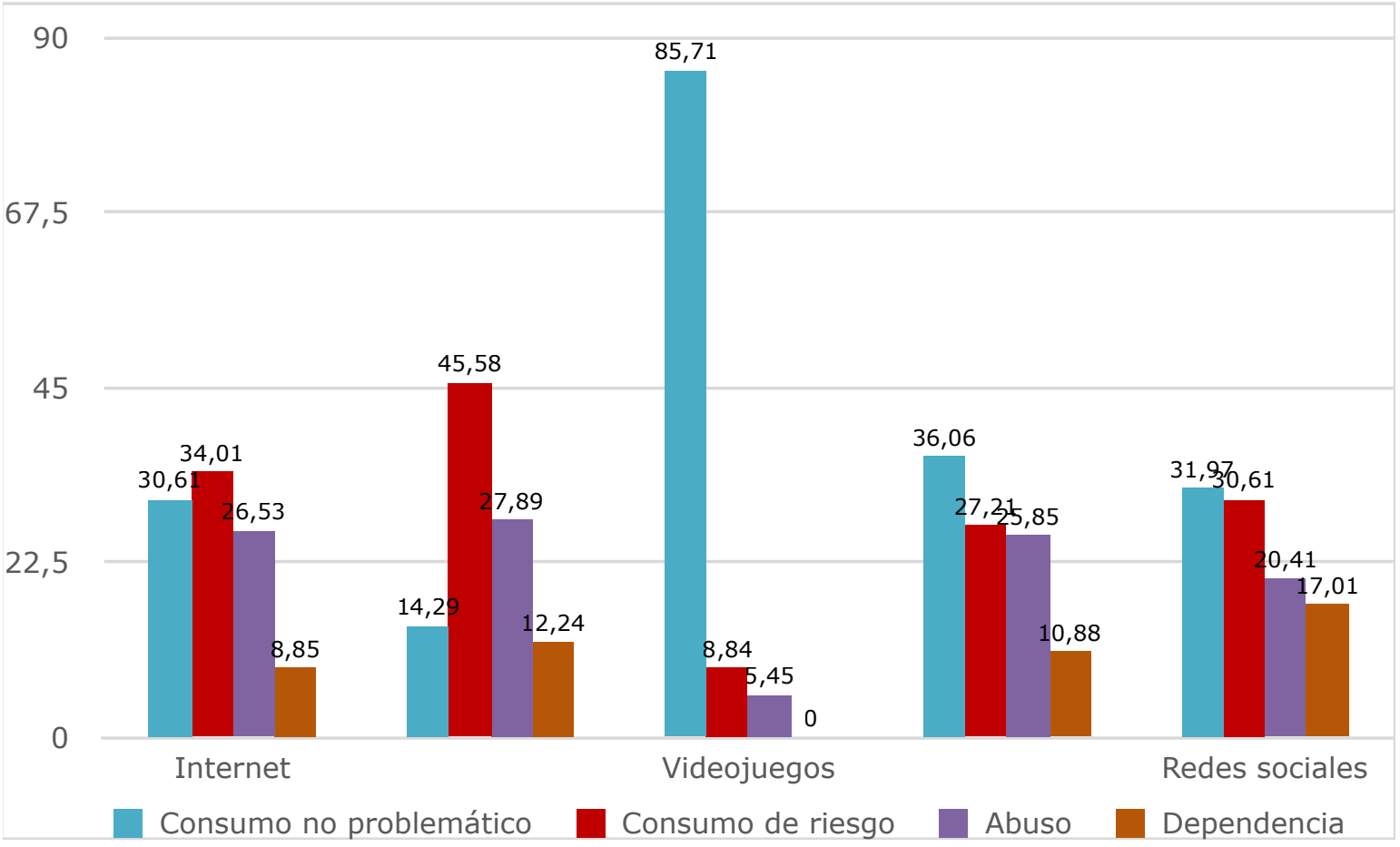

Figura. 1. Respuestas al MULTICAGE-TIC en el pre-test.

A nivel general, el alumnado refleja un mayor porcentaje de casos asociados al consumo no problemático o de riesgo que de abuso o dependencia. Así, el nivel problemático de abuso o dependencia oscila entre el $40.13 \%$ en el caso de los móviles y el $5.45 \%$ en los videojuegos, siendo siempre inferior a la mitad de los casos. Por el contrario, en el pre-test, los consumos no problemáticos representan a 1 de cada 3 estudiantes, salvo en el caso de los móviles (14.29\%) y videojuegos $(85.71 \%)$ que reflejan el mínimo y máximo porcentaje respecto al consumo no problemático.

Atendiendo específicamente a cada escala, encontramos que en el patrón de uso de internet predomina la conducta de riesgo $(34.01 \%)$, siendo algo inferior el porcentaje de estudiantes que realizan un consumo no problemático (30.61). Resaltar que 1 de cada 4 estudiantes ya manifiestan un uso abusivo de internet (26.53\%) antes de la pandemia, mostrando dependencia solo el $8.85 \%$ de los encuestados. En cuanto al uso del móvil, nos encontramos con la TIC que tiene un mayor consumo de riesgo $(45.58 \%)$ y de abuso de su utilización $(27.89 \%)$ en el pre-test. Por igual, es significativo que estemos ante el elemento tecnológico que refleja menor porcentaje de consumo no problemático $(14.29 \%$ ), de forma que un $85.71 \%$ de los participantes presentan problemas (en distinto nivel) derivados de su utilización. Por su parte, los videojuegos tienen el mayor porcentaje de consumo de no riesgo $(85.71 \%)$, siendo bajo el porcentaje de alumnado con conductas problemáticas $(14.29 \%)$, destacando que ningún participante $(0 \%)$ presenta dependencia en relación a su uso. Respecto a la mensajería instantánea, el mayor porcentaje refleja un consumo no problemático $(36.06 \%)$, destacando la semejanza entre los que presentan conductas de riesgo $(27.21 \%)$ y abuso (25.85), existiendo dependencia en 1 de cada 10 estudiantes (10.88\%). Finalizamos con las redes sociales, siendo el elemento TIC que mayor nivel de dependencia presenta en el pre-test $(17.01 \%)$, subrayando así una mayor adicción al uso de redes sociales entre los participantes del estudio. Los datos de uso no problemático (31.97\%) y de riesgo (30.61\%) son los más semejantes, con apenas $1.36 \%$ de diferencia.

Pasamos a los resultados del post-test (Figura 2), en pleno estado de alarma y confinamiento. 


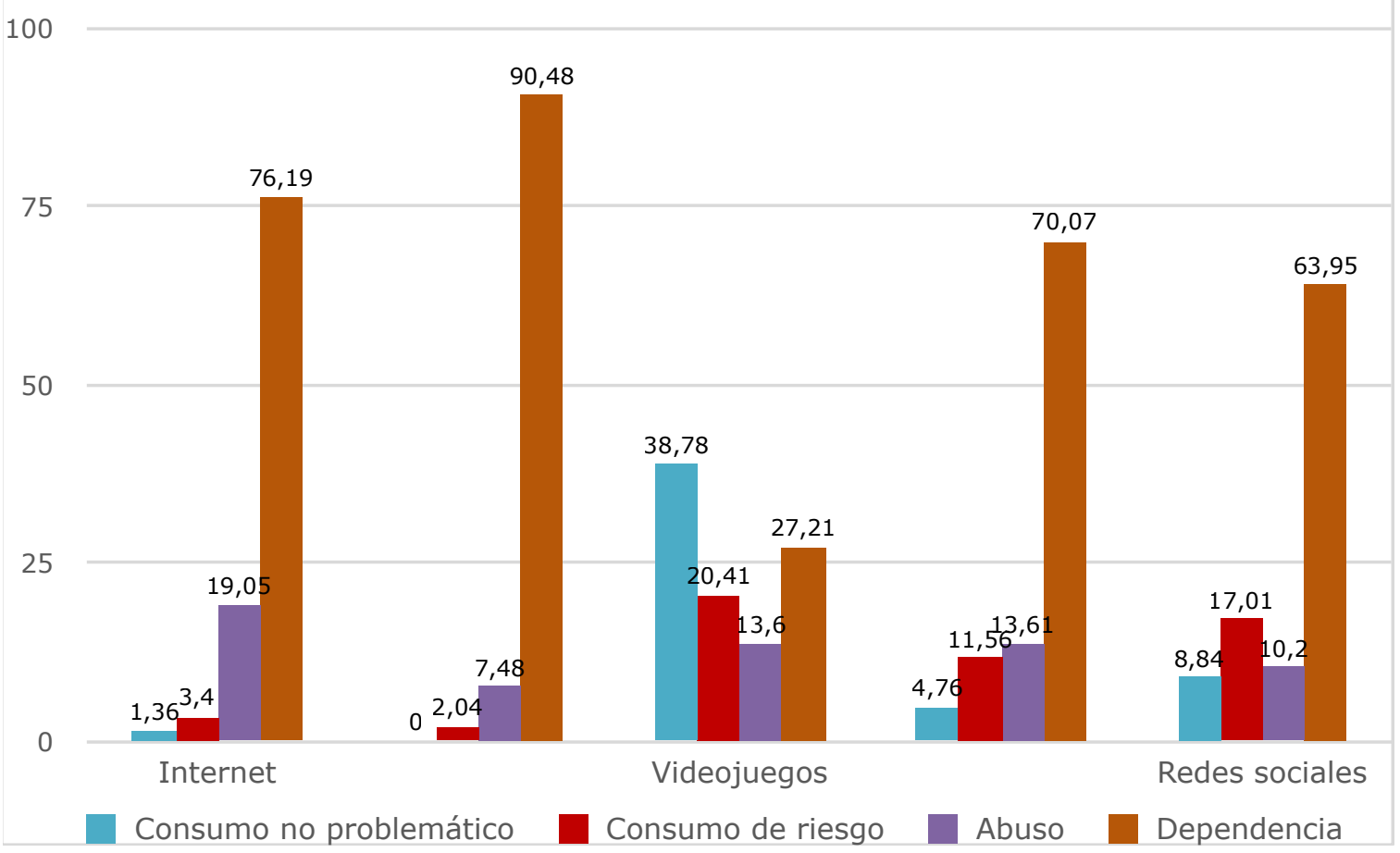

Figura. 2. Respuestas al MULTICAGE-TIC en el post-test.

Como podemos observar, la dependencia hacia las TIC se convierte en mayoritaria en todas las escalas, salvo en videojuegos. Una conducta de dependencia que afecta a 9 de cada 10 estudiantes respecto al uso del móvil $(90.48 \%)$, el mayor porcentaje en el post-test, mientras que en los videojuegos se aproxima a los 3 estudiantes de cada 10 (27.21\%). Igualmente, sin contar a los videojuegos, es significativo que el porcentaje de estudiantes que presentan un consumo no problemático en las diferentes escalas, se sitúe por debajo del $10 \%$, destacando nuevamente el móvil donde ningún participante considera que su forma de utilizarlo sea no problemática.

Si describimos cada escala de forma pormenorizada, encontramos que internet, durante el confinamiento, es utilizado de forma abusiva por 2 de cada 10 estudiantes (19.05\%), generando dependencia en el $76.19 \%$ de los participantes. De este modo, incluso las conductas de riesgos tienen porcentajes poco significativos (3.4\%), siendo ínfima si nos referimos al uso no problemático $(1.36 \%)$. La dependencia hacia el uso del móvil, como ya hemos señalado, es muy alta. Junto con el uso abusivo, representan al $97.98 \%$ de los participantes, por lo que podemos destacar un problema real respecto a la utilización del móvil durante la pandemia del que los participantes son conscientes, a tenor de los resultados alcanzados. Respecto a los videojuegos, encontramos una subida considerable en todos los niveles, hecho derivado de que su uso no problemático pasa del $85.71 \%$ antes del confinamiento al $38.78 \%$ durante el mismo. Destacar la presencia en el post-test de conductas de dependencia $(27.21 \%)$, de forma que junto a los participantes que manifiestan un uso abusivo (13.60\%), tiene como resultado que 4 de cada 10 estudiantes utilizan de forma problemática los videojuegos. En cuanto a la mensajería instantánea, 7 de cada 10 participantes señalan tener dependencia hacia este tipo de aplicaciones durante el confinamiento, siendo solo un $4.76 \%$ los que no manifiestan una conducta problemática al usarla. Para cerrar, las redes sociales han aumentado el porcentaje de estudiantes que presenta dependencia hasta el $63.95 \%$. Sin embargo, pese al crecimiento, ha pasado de ser la escala con mayor nivel de dependencia en el pre-test a la penúltima en el post-test. No obstante, como en el resto de elementos TIC, el porcentaje de estudiantes que realizan un uso no considerado como problemático es bajo $(8.84 \%)$. 
A continuación, comentaremos con más detenimiento la evolución de los resultados entre el pretest y el post-test por escalas (Figura 3 ).

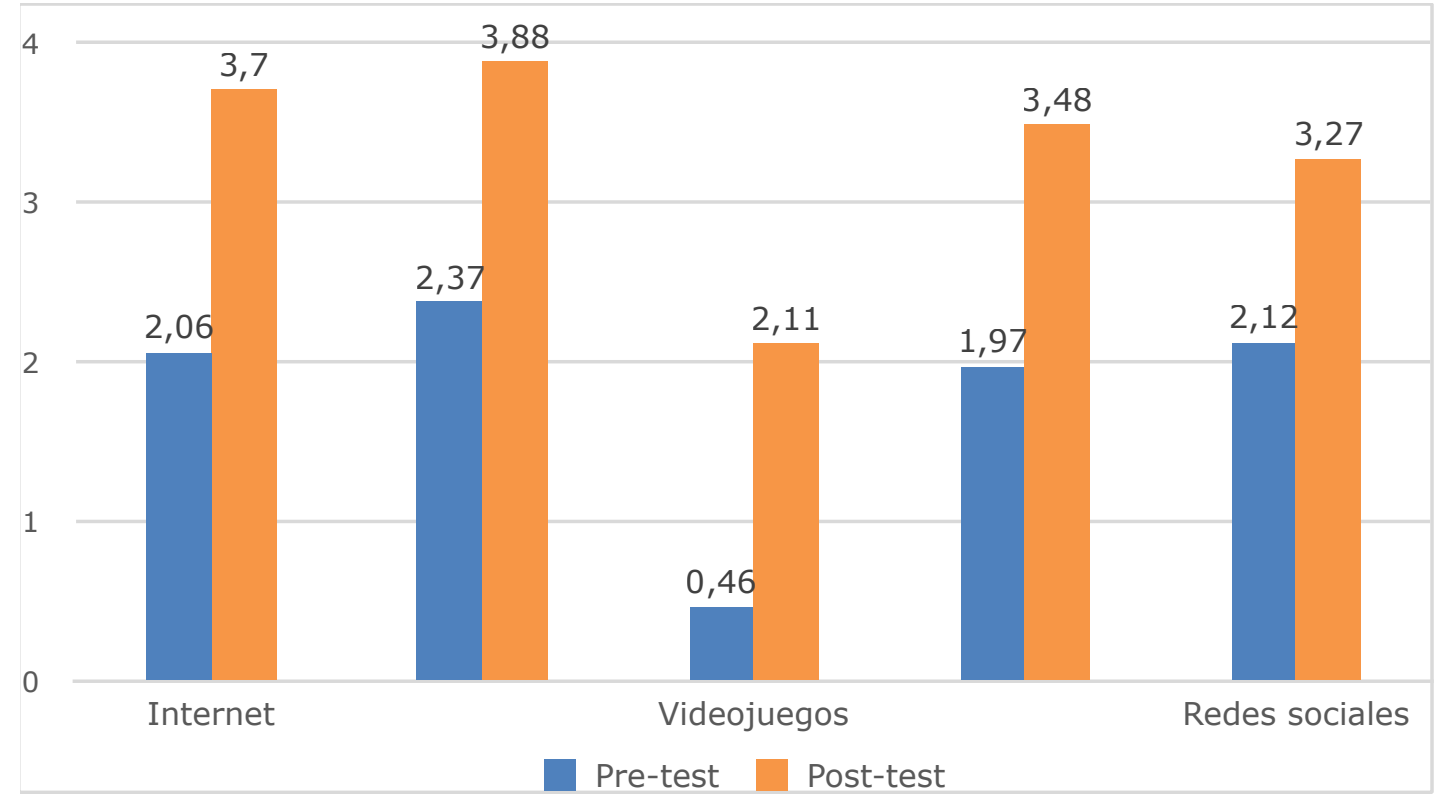

Figura. 3. Comparativa pre-test / post-test por escalas.

Los resultados del pre-test, respecto al uso de las TIC, reflejan una media de respuestas que se sitúa en un consumo de riesgo, aproximándose en el caso del móvil al abuso. Por el contrario, la utilización de los videojuegos no es problemática. Sin embargo, el estado de alarma y confinamiento ha conllevado un aumento manifiesto del uso de todas las TIC, pasando a un uso dependiente en todas las categorías, excepto en los videojuegos, donde se sitúan en una conducta de riesgo próxima al abuso. La gráfica evidencia como antes del confinamiento, ya existían algunas conductas y usos de las TIC que podrían convertirse en problemáticas. La situación excepcional de aislamiento y reclusión vividas durante el COVID-19, con el consiguiente incremento de las interacciones virtuales a nivel personal y profesional, han generado un contexto propicio para que dichos comportamientos problemáticos, en cuanto a la utilización de las TIC, hayan aumentado hasta ser un problema latente.

Después del análisis exploratorio entre el pre-test y el post-test, vamos a comprobar si las diferencias observadas son estadísticamente significativas. Para ello, como adelantamos en el apartado de análisis de datos, aplicamos la prueba no paramétrica W de Wilcoxon para comparar las medias de las escalas mediante estadística inferencial (Tabla 5). 


\begin{tabular}{|c|c|c|c|c|c|}
\hline \multirow{2}{*}{ Elemento } & \multirow{2}{*}{ Momento } & \multirow{2}{*}{ Media } & \multirow{2}{*}{ Desv. Típica } & \multicolumn{2}{|c|}{ Wilcoxon } \\
\hline & & & & Z & $p$ \\
\hline \multirow{2}{*}{ Internet } & Pre-test & 2.06 & 1.07 & \multirow{2}{*}{-9.987} & \multirow[b]{2}{*}{$0.000 *$} \\
\hline & Post-test & 3.70 & 0.60 & & \\
\hline \multirow{2}{*}{ Móvil } & Pre-test & 2.37 & 0.91 & \multirow{2}{*}{-9.927} & \multirow{2}{*}{$0.000 *$} \\
\hline & Post-test & 3.88 & 0.38 & & \\
\hline \multirow{2}{*}{ Videojuegos } & Pre-test & 0.46 & 0.87 & \multirow{2}{*}{-9.359} & \multirow{2}{*}{$0.000 *$} \\
\hline & Post-test & 2.11 & 1.47 & & \\
\hline \multirow{2}{*}{$\begin{array}{l}\text { Mensajería } \\
\text { instantánea }\end{array}$} & Pre-test & 1.97 & 1.23 & \multirow{2}{*}{-9.322} & \multirow{2}{*}{$0.000 *$} \\
\hline & Post-test & 3.48 & 0.90 & & \\
\hline \multirow{2}{*}{ Redes sociales } & Pre-test & 2.12 & 1.24 & \multirow[b]{2}{*}{-7.715} & \multirow{2}{*}{$0.000 *$} \\
\hline & Post-test & 3.27 & 1.11 & & \\
\hline
\end{tabular}

Tabla 5. Resultados de la prueba W de Wilcoxon. $*=p<.05$

Los resultados corroboran lo observado en el análisis exploratorio, confirmando la existencia de diferencias significativas entre el pre-test y el post-test en todas las escalas. De este modo, el uso y abuso de las TIC por parte de los profesionales de la educación en formación ha aumentado durante el periodo de confinamiento causado por la COVID-19.

\section{Discusión}

Este estudio nos ha permitido conocer mejor la realidad de nuestros estudiantes universitarios en torno al uso de determinados recursos y aplicaciones tecnológicas. Los datos obtenidos nos han ayudado a definir un contexto inicial que se ha visto alterado por la situación de pandemia mundial derivada del COVID-19 y, en concreto, por los periodos de confinamiento, restricciones de la movilidad y aislamiento social derivados de las políticas y conductas cívicas en favor de la prevención.

Sin duda, la variable del COVID-19 ha alterado la vida en todos los ámbitos. Ya hay evidencia científica que correlaciona la situación socio-sanitaria derivada de la pandemia con el agravamiento de diferentes adicciones (Columb et al., 2020; Dubey et al., 2020; Håkansson et al., 2020). El incremento del hábito de uso respecto a la tecnología no es ajeno a la influencia de la pandemia (Dong et al., 2020; Wang et al., 2020). Por ello, estableceremos una doble línea de discusión; la primera, centrada en una normalidad previa a la que aspiramos retornar; y la segunda, sobre la influencia de la pandemia en los ámbitos estudiados, que dan cuenta del estado actual de la situación y del trabajo que se cierne en el horizonte para revertir las consecuencias del COVID-19 sobre la forma en la que nuestros estudiantes se relacionan con la tecnología.

Respecto a la realidad previa a la pandemia, los resultados que hemos obtenido apuntan hacia patrones de uso estables asociados a un consumo no problemático de los dispositivos de acceso a internet y las aplicaciones analizadas (videojuegos, mensajería instantánea y redes sociales). El teléfono móvil es el dispositivo que utilizan de manera más frecuente los estudiantes universitarios. A este dispositivo se vinculan los niveles más altos en las categorías de "Consumo de riesgo" y "Abuso". Este hallazgo se alinea con los realizados en estudios previos (Lepp et al., 2014; Miri 
et al., 2020; Walsh et al., 2011). Podemos concluir que el teléfono móvil, y en concreto, el Smartphone, es un dispositivo muy utilizado por los estudiantes universitarios en nuestro contexto, y que ese gran consumo tiene asociado un riesgo de dependencia.

Otro de los hallazgos significativos que hemos realizado tiene que ver con el uso de los videojuegos. En contra de lo que pensábamos inicialmente, los universitarios no presentan un gran consumo de video-juegos. Reportan el valor más alejado de la adicción. Esto respalda la conclusión de Entwistle et al. (2020), que sostienen que los vídeojuegos no son intrínsecamente adictivos.

Más que al juego, el uso del móvil entre universitarios se relaciona con la comunicación a través de programas de mensajería instantánea y, por encima de ello, para publicar contenido e interaccionar en redes sociales. De las posibilidades de uso que hemos estudiado, las redes sociales son las que presentan valores más altos en las categorías de "consumo de riesgo", "abuso" y "dependencia". Como apuntábamos en la introducción, el diseño de las aplicaciones de redes sociales presenta elementos específicos enfocados a fidelizar un uso continuo. Consideramos que este particular, unido a las características psicológicas propias de este rango de edad, están detrás de los altos niveles de uso que presentan en los universitarios estudiados. Otras investigaciones sobre adicción a redes sociales en poblaciones universitarias apuntan en esta misma dirección (Aparicio-Martínez et al., 2020; Foerster et al., 2015). Frente a ellas, el trabajo de Robles y Dany (2016) no encuentra usos inadecuados en su contexto de referencia.

No podemos descartar que los valores elevados de uso de dispositivos móviles se deriven de una adicción a las redes sociales, como ya advirtieron Jasso Medrano et al. (2017, p. 2836):

El uso problemático del móvil tuvo una relación alta con la adicción a redes sociales, además de que la frecuencia de uso del teléfono móvil se relacionó un poco más con la conducta adictiva a las redes sociales que con el uso problemático de este, coincidiendo con la postura de que el uso problemático del móvil podría ir más encaminado por la conducta adictiva a las redes sociales que por la dependencia del dispositivo en sí, siendo un medio de acceso.

En esta línea, el uso excesivo del móvil podría ser considerado como un factor de riesgo y un indicador de adicción a redes sociales (Echeburúa, \& Corral, 2010; Rosales, \& Medrano, 2019). Sin duda, y pese a existir evidencia en este sentido, consideramos que se trata de una cuestión sobre la que se debería profundizar para confirmarla.

Con la pandemia y las restricciones de movilidad y de contacto social, el consumo de internet, telefonía móvil, redes sociales, videojuegos y mensajería instantánea incrementaron de manera muy notable. Los datos recogidos en la fase más restrictiva de las medidas de prevención social de contagio mostraron valores de "dependencia" vinculados a Internet, el móvil, redes sociales y mensajería instantánea. En todos los casos con un incremento muy acusado respecto a los valores previos a la situación de alerta sanitaria. Esto nos lleva a concluir que el aislamiento social causado por las medidas de prevención ha incrementado el consumo individual de los universitarios respecto a los dispositivos y aplicaciones analizadas. En la actualidad, el nivel de consumo más generalizado se corresponde con patrones de adicción. El estudio de Siste et al. (2020) también identifica un incremento de consumo asociado a la pandemia, sin embargo no asocia a ella un mayor riesgo de adicción al internet. 


\section{Referencias}

Ahmadi, K. (2014). Internet addiction among Iranian adolescents: a nationwide study. Acta Medica Iranica, $52(6), 467-472$.

Aparicio-Martínez, P., Ruiz-Rubio, M., Perea-Moreno, A.-J., Martínez-Jiménez, M. P., Pagliari, C., Redel-Macías, M. D., \& Vaquero-Abellán, M. (2020). Gender differences in the addiction to social networks in the Southern Spanish university students. Telematics and Informatics, 46, 101304. https://doi.org/ 101304. 10.1016/j.tele.2019.101304

Asociación Española de Videojuegos (AEVI) (2019). La industria del videojuego en España. Anuario 2019. AEVI. http://www.aevi.org.es/web/wp-content/uploads/2020/04/AEVI-ANUARIO-2019.pdf

Błachnio, A., Przepiórka, A., \& Pantic, I. (2015). Internet Use, Facebook Intrusion, and Depression: Results of a Cross-Sectional Study. European Psychiatry, 30(6), 681-684. https://doi.org/10.1016/ j.eurpsy.2015.04.002

Brennan, A., \& Dempsey, M. (2018). The student voice: the students own views on smartphone usage and impact on their academic performance. 12th International Technology, Education and Development Conference, 5-7 de marzo, 2018, Valencia. https://doi.org/10.21125/inted.2018.1836

Carbonell, X., Chamarro, A., Griffiths, M., Oberst, U., Cladellas, R., \& Talarn, A. (2012). Problematic Internet and cell phone use in Spanish teenagers and young students. Anales de Psicología, 28, 789-796.

Catalina, B., García, A., \& Montes, M. (2015). Jóvenes y consumo de noticias a través de Internet y los medios sociales. Historia y Comunicación Social, 20(2), 601-619. http://dx.doi.org/10.5209/ rev_HICS.2015.v20.n2.51402

Cho, H., Kwon, M., Choi, J.-H., Lee, S.-K., Choi, J. S., Choi, S.-W., \& Kim, D.-J. (2014). Development of the Internet addiction scale based on the Internet Gaming Disorder criteria suggested in DSM-5. Addictive Behaviors, 39(9), 1361-1366. https://doi.org/10.1016/j.addbeh.2014.01.020

Columb, D., Hussain, R., \& O'Gara, C. (2020). Addiction psychiatry and COVID-19: Impact on patients and service provision. Irish Journal of Psychological Medicine, 37(3), 164-168. https://doi.org/10.1017/ ipm.2020.47

Dhalla, S., \& Kopec, J. A. (2007). The CAGE questionnaire for alcohol misuse: a review of reliability and validity studies. Clinical \& Investigative Medicine, 30, 33-41. https://doi.org/10.25011/cim.v30i1.447

De la Villa Moral, M., \& Suárez, C. (2016). Factores de riesgo en el uso problemático de Internet y del teléfono móvil en adolescentes españoles. Revista Iberoamericana de Psicología y Salud, 7(2), 69-78. https:// doi.org/10.1016/j.rips.2016.03.001

Devine, P., \& Lloyd, K. (2012). Internet use and psychological well-being among 10-year-old and 11-year-old children. Child Care in Practice, 18(1), 5-22. https://doi.org/10.1080/13575279.2011.621888DovalAvendaño,

Dong, H., Yang, F., Lu, X., \& Hao, W. (2020). Internet Addiction and Related Psychological Factors Among Children and Adolescents in China During the Coronavirus Disease 2019 (COVID-19) Epidemic. Frontiers in Psychiatry, 11, 751. https://doi.org/10.3389/fpsyt.2020.00751

Dubey, M. J., Ghosh, R., Chatterjee, S., Biswas, P., Chatterjee, S., \& Dubey, S. (2020). COVID-19 and addiction. Diabetes \& Metabolic Syndrome, 14(5), 817-823. https://doi.org/10.1016/j.dsx.2020.06.008

Echeburúa, E., \& Corral, P. de. (2010). Adicción a las nuevas tecnologías y a las redes sociales en jóvenes: Un nuevo reto. Adicciones, 22(2), 91-96. https://doi.org/10.20882/adicciones.196

Elhai, J. D., Dvorak, R. D., Levine, J. C., \& Hall, B. J. (2017). Problematic smartphone use: A conceptual overview and systematic review of relations. J Affect Disord, 1, 207-251. https://doi.org/10.1016/ j.jad.2016.08.030

Entwistle, G. J. M., Blaszczynski, A., \& Gainsbury, S. M. (2020). Are video games intrinsically addictive? An international online survey. Computers in Human Behavior, 112, 106464. https://doi.org/10.1016/ j.chb.2020.106464 
Estévez, A., Herrero-Fernández, D., Sarabia, I., \& Jáuregui, P. (2015). The impulsivity and sensation-seeking mediators of the psychological consequences of pathological gambling in adolescence. Journal of Gambling Studies, 31, 91-103. https://doi.org/10.1007/s10899-013-9419-0.

Fernández Álvarez, Y., \& Fajardo, M. (2020, noviembre 19). Tecnología versus libertad [Programa de radio]. A golpe de bit. Radio Nacional de España, RNE. https://bit.ly/3lh850x

Foerster, M., Roser, K., Schoeni, A., \& Röösli, M. (2015). Problematic mobile phone use in adolescents: Derivation of a short scale MPPUS-10. International Journal of Public Health, 60(2), 277-286. https:// doi.org/10.1007/s00038-015-0660-4

García-Jiménez, A., Tur-Viñes, V., \& Pastor Ruiz, Y. (2018). Consumo mediático de adolescentes y jóvenes. Icono 14, 16(1), 22-46. https://doi.org/10.7195/ri14.v16i1.1101

Garrido-Elustondo, S., Reneses, B., Navalón, A., Martín, O., Ramos, I., \& Fuentes, M. (2016). Capacidad de detección de patología psiquiátrica por el médico de familia. Atención Primaria, 48, 449-457. https:// doi.org/10.1016/j.aprim.2015.09.009.

González-Cortés, E., Córdoba-Cabús, A., \& Gómez, M. (2020). Una semana sin smartphone: usos, abuso y dependencia del teléfono móvil en jóvenes. Bordón. Revista De Pedagogía, 72(3), 105-121. https:// doi.org/10.13042/Bordon.2020.79296

Griffiths, M. D., Kuss, D. J., \& Demetrovics, Z. (2014). Social Networking Addiction An Overview of Preliminary Findings. In K. Paul \& L. Curtiss (Eds.), Behavioral Addictions (pp. 119-141). Elsevier. https://doi.org/ 10.1016/B978-0-12-407724-9.00006-9

Håkansson, A., Fernández-Aranda, F., Menchón, J. M., Potenza, M. N., \& Jiménez-Murcia, S. (2020). Gambling during the COVID-19 crisis-A cause for concern? Journal of Addiction Medicine, 14(4), e10-e12. https://doi.org/10.1097/ADM.0000000000000690

Hernández, E. (2020, abril 19). Las videollamadas y los mensajes no pueden suplir el contacto personal durante el confinamiento [Television]. Telediario. Radio Televisión Española, RTVE. https://bit.ly/ $3 \mathrm{mjNc6C}$

Jasso Medrano, J. L., López Rosales, F., \& Díaz Loving, R. (2017). Conducta adictiva a las redes sociales y su relación con el uso problemático del móvil. Acta de Investigación Psicológica, 7(3), 2832-2838. https://doi.org/10.1016/j.aipprr.2017.11.001

Instituto Nacional de Estadística. (2020). Encuesta sobre Equipamiento y Uso de Tecnologías de Información y Comunicación en los Hogares. Año 2020. https://bit.ly/2HLvPwn

Lepp, A., Barkley, J. E., \& Karpinski, A. C. (2014). The relationship between cell phone use, academic performance, anxiety, and Satisfaction with Life in college students. Computers in Human Behavior, 31, 343-350. https://doi.org/10.1016/j.chb.2013.10.049

Martín Del Pozo, M. (2015). Videojuegos y aprendizaje colaborativo. Experiencias en torno a la etapa de Educación Primaria. Education in the Knowledge Society (EKS), 16(2), 69-89. https://doi.org/ 10.14201/eks20151626989

Méndez-Gago, S., \& González-Robledo, L. (2018). Uso y abuso de las Tecnologías de la Información y la Comunicación por adolescentes. Un estudio representativo de la ciudad de Madrid. Universidad Camilo José Cela.

Miri, M., Tiyuri, A., Bahlgerdi, M., Miri, M., Miri, F., \& Salehiniya, H. (2020). Mobile addiction and its relationship with quality of life in medical students. Clinical Epidemiology and Global Health, 8(1), 229-232. https://doi.org/10.1016/j.cegh.2019.08.004

Navas, J. F., Torres, A., Cándido, A., \& Perales, J. C. (2014). ¿'Nada' o 'un poco'? ¿'Mucho' o 'demasiado'? La impulsividad como marcador de gravedad en niveles problemático y no problemático de uso de alcohol e Internet. Adicciones, 26, 146-158. https://doi.org/10.20882/adicciones.19

Organización Mundial de la Salud (Ed.). (2019). Hacia una mejor delimitación del trastorno por uso de videojuegos. Boletín de la Organización Mundial de la Salud, 97(6), 382-383. https://doi.org/10.2471/ 


\section{BLT.19.020619}

Orlowski, J. (2020, septiembre 9). The Social Dilemma [Documentary, Drama]. Exposure Labs, Argent Pictures, The Space Program.

Orte Socías, C., Ballester Brage, L., \& Nevot-Caldentey, L. (2020). Family support against COVID-19. https:// doi.org/10.1590/SciELOPreprints.297

Ostovar, S., Allahyar, N., Aminpoor, H., Moafian, F., Nor, M. B. M., \& Griffiths, M. D. (2016). Internet addiction and its psychosocial risks (depression, anxiety, stress and loneliness) among Iranian adolescents and young adults: A structural equation model in a cross-sectional study. International Journal of Mental Health and Addiction, 14(3), 257-267. https://doi.org/10.1007/s11469-015-9628-0

Paricio del Castillo, R., \& Pando Velasco, M. F. (2020). Salud mental infanto-juvenil y pandemia de Covid-19 en España: Cuestiones y retos. Revista de Psiquiatría Infanto-Juvenil, 37(2), 30-44. https://doi.org/ 10.31766/revpsij.v37n2a4

Pedrero-Pérez, E. J., Rodríguez-Monje, M. T., Gallardo-Alonso, F., Fernández-Girón, M., Pérez-López, M., \& Chicharro-Romero, J. (2007). Validación de un instrumento para la detección de trastornos de control de impulsos y adicciones: el MULTICAGE CAD-4. Trastornos Adictivos, 9, 269-278. https://doi.org/ 10.1016/S1575-0973(07)75656-8.

Pedrero-Pérez, E.J., Ruiz-Sánchez, J.M., Rojo-Mota, G., Llanero-Luque, M., Pedrero-Aguilar, J., Morales-Alonso, S., \& Puerta-García, C. (2018). Tecnologías de la Información y la Comunicación (TIC): uso problemático de Internet, videojuegos, teléfonos móviles, mensajería instantánea y redes sociales mediante el MULTICAGE-TIC. Adicciones, 30(1), 19-32. https://doi.org/10.20882/adicciones.806

Real Decreto 463/2020, de 14 de marzo, por el que se declara el estado de alarma para la gestión de la situación de crisis sanitaria ocasionada por el COVID-19. Boletín Oficial del Estado, 67, p. 1-15. https://bit.ly/2JbQcDU

Revuelta, F. I., \& Esnaola, G. A. (2013). Videojuegos en redes sociales: Perspectivas del edutainment y la Pedagogía lúdica en el aula. Laertes educación.

Robles, A., \& Dany, E. (2016). Indicadores de adicción a las redes sociales en universitarios de Lima. Revista Digital de Investigación en Docencia Universitaria, 10(2), 48-58. https://doi.org/10.19083/ridu.10.494

Rodríguez-Monje, M.T., Pedrero-Pérez, E. J., Rodríguez-Alonso, E., Fernández-Girón, M., Pastor-Ramos, V., Mateo-Madurga, A., Cura-González, I., Sanz-Cuesta, T., Arana-Cañedo, C., Sánchez-Salgado, M.P., \& Escriva-Ferrairo, R.A. (2019). MULTICAGE CAD-4 for behavioral addiction screening: structural validity after inclusion of a scale on smartphone abuse. Anales de Psicología, 35(1), 41-46. https://dx.doi.org/ 10.6018/analesps.35.1.324311

Rosales, F. L., \& Jasso Medrano, J. L. (2019). Interacción entre el uso y la adicción a las redes sociales y teléfonos móviles entre estudiantes universitarios. Revista de Investigación en Tecnologías de la Información, 7(14), 76-88. https://doi.org/10.36825/RITI.07.14.007

Ruiz-Palmero, J., Sánchez-Rivas, E., Gómez-García, M., \& Sánchez Vega, E. (2019). Future Teachers' Smartphone Uses and Dependence. Education Sciences, 9(3), e194. https://doi.org/10.3390/ educsci9030194

Ruiz-Palmero, J., Sánchez-Rodríguez, J., \& Trujillo-Torres, J. M. (2016). Utilización de Internet y dependencia a teléfonos móviles en adolescentes. Revista Latinoamericana de Ciencias Sociales, Niñez y Juventud, 14(2), 1357-1369.

Sánchez-Rivas, E., Ruiz-Palmero, J., \& Sánchez-Rodríguez, J. (2017). Videojuegos frente a fichas impresas en la intervención didáctica con alumnado con necesidades educativas especiales. Educar, 53(1), 29-48. https://doi.org/10.5565/rev/educar.844

Siste, K., Hanafi, E., Sen, L. T., Christian, H., Adrian, Siswidiani, L. P., Prabowo, A., Julivia, B., \& Suwartono, C. (2020). The Impact of Physical Distancing and Associated Factors Towards Internet Addiction Among Adults in Indonesia During COVID-19 Pandemic: A Nationwide Web-Based Study. Frontiers in Psychiatry, 11, 924. https://doi.org/10.3389/fpsyt.2020.580977 
Tsitsika, A., Tzavela, E., Mavromati, F., \& EU NET ADB Consortium. (2013). Investigación sobre conductas adictivas a Internet entre los adolescentes europeos. http://www.injuve.es/sites/default/files/2013/03/ publicaciones/FinalResearchInternet-ES.pdf

Viñals Blanco, A., Abad Galzacorta, M., \& Aguilar Gutiérrez, E. (2014). Jóvenes conectados: Una aproximación al ocio digital de los jóvenes españoles. Communication Papers -Media Literacy \& Gender Studies-, $3(4), 52-68$.

Walsh, S. P., White, K. M., Cox, S., \& Young, R. McD. (2011). Keeping in constant touch: The predictors of young Australians' mobile phone involvement. Computers in Human Behavior, 27(1), 333-342. https://doi.org/10.1016/j.chb.2010.08.011

Wang, Y.-Y., Long, J., Liu, Y.-H., Liu, T.-Q., \& Billieux, J. (2020). Factor structure and measurement invariance of the problematic mobile phone use questionnaire-short version across gender in Chinese adolescents and young adults. BMC Psychiatry, 20(1), 34. https://doi.org/10.1186/s12888-020-2449-0 